\title{
TITLE:
}

\section{Control of photofragment velocity anisotropy by optical alignment of $\mathrm{CH} 3 \mathrm{I}$}

\section{AUTHOR(S):}

Sugita, A; Mashino, M; Kawasaki, M; Matsumi, Y; Gordon, RJ; Bersohn, R

\section{CITATION:}

Sugita, A ...[et al]. Control of photofragment velocity anisotropy by optical alignment of CH3I. JOURNAL OF CHEMICAL PHYSICS 2000, 112(5): 2164-2167

\section{ISSUE DATE:}

2000-02-01

URL:

http://hdl.handle.net/2433/39723

\section{RIGHT:}

Copyright 2000 American Institute of Physics. This article may be downloaded for personal use only. Any other use requires prior permission of the author and the American Institute of Physics. 


\title{
Control of photofragment velocity anisotropy by optical alignment of $\mathrm{CH}_{3} \mathrm{I}$
}

\author{
Akihiro Sugita, Michio Mashino, and Masahiro Kawasaki \\ Department of Molecular Engineering, Kyoto University, Kyoto 606-8501, Japan \\ Yutaka Matsumi \\ Solar Terrestrial Environment Laboratory, Nagoya University, Toyokawa 442-8507, Japan \\ Robert J. Gordon \\ Department of Chemistry, University of Illinois, Chicago, Illinois 60607-7061 \\ Richard Bersohn \\ Department of Chemistry, Columbia University, New York, New York 10027
}

(Received 20 August 1999; accepted 26 October 1999)

\begin{abstract}
A molecule placed in an electric field can be aligned because of the anisotropy of its polarizability tensor. As an alternative to high static electric fields, the electric field of a pulsed laser can be used for alignment. The alignment can be demonstrated by measurement of the control of the anisotropy of photodissociated fragments generated by polarized light. Sakai et al. have recently reported such an experiment on aligned iodine molecules [J. Chem. Phys. 110, 10235 (1999)]. This paper reports an independent finding of the same phenomenon with $\mathrm{CH}_{3} \mathrm{I}$. General equations are presented for the degree of alignment and its control of the photodissociation amplitude. The control parameter for a symmetric top or linear molecule is $-\left(\alpha-\alpha_{z z}\right)\left(E^{2} / 2 k T\right) P_{2}(\cos \chi)$, where $\alpha$ is the mean polarizability, $\alpha_{z z}$ is the element of the polarizability tensor along the symmetry axis, and $\chi$ is the angle between the $E$ vectors of the aligning and dissociating lasers. The fragment velocity anisotropy can be increased or decreased by varying this control parameter. (C) 2000 American Institute of Physics. [S0021-9606(00)00604-8]
\end{abstract}

\section{INTRODUCTION}

A freely rotating molecule placed in a weak angularly dependent potential has perturbed energy levels and wave functions. Because of the torque exerted during its rotation, angular momentum is no longer a constant of the motion. As the angularly dependent potential is increased, the angular distribution of the molecule becomes anisotropic. The molecule, classically speaking, spends more time in the vicinity of the potential minimum even though it is not trapped.

Recently, there has been increasing interest in controlling the rotation of gas phase molecules by external electric fields. ${ }^{1-10}$ A necessary condition for appreciable alignment is that $\mu E$ should be comparable to $k T$, where $\mu$ is the electric dipole moment of the molecule, and $E$ is the optical electric field. Assuming $\mu=1 \mathrm{D}\left(10^{-18} \mathrm{esu} \mathrm{cm}\right)$ and $T=300 \mathrm{~K}$, a field of $1 \times 10^{7} \mathrm{~V} / \mathrm{cm}$ would be required. Such static fields are impractical, in part because they produce discharges in a gas. It has been shown that this difficulty can be partially overcome by the use of a molecular beam cooled by nozzle expansion. ${ }^{2}$ At a temperature of $3 \mathrm{~K}$ the required field would be $10^{5} \mathrm{~V} / \mathrm{cm}$, large but attainable. The brute force method, which orients the molecular dipole moment along the electric field, is obviously limited to polar molecules. ${ }^{1}$ Friedrich and Herschbach $^{3}$ have pointed out that the use of an optical field offers alternative possibilities. Optical frequencies are much higher than rotational frequencies; for example, $1064 \mathrm{~nm}$ light has a period of $3.5 \mathrm{fs}$. The relatively slowly rotating molecule is unable to follow the rapidly fluctuating electric field, and the first order effect averages to zero. However, the second order perturbation due to the polarizability anisotropy of the molecule may be appreciable.

Sakai et al. ${ }^{9}$ have reported the photodissociation of iodine molecules with a strong $\left(1.4 \times 10^{12} \mathrm{~W} / \mathrm{cm}^{2}\right)$ IR laser field used for alignment. Using a sample of aligned iodine molecules, Larsen et al. ${ }^{10}$ showed control of the photofragment branching ratios of the $\mathrm{I}\left({ }^{2} P_{3 / 2}\right)$ and $\mathrm{I}\left({ }^{2} P_{1 / 2}\right)$ channels. We have independently observed the alignment phenomenon in methyl iodide. This paper reports the experimental results as well as a general theoretical expression for the alignment and its control of the photofragment angular distribution. The angular distribution of $\mathrm{I}\left({ }^{2} P_{3 / 2}\right)$ atoms from the photodissociation of $\mathrm{CH}_{3} \mathrm{I}$ at $304 \mathrm{~nm}$ was measured with a photofragment imaging apparatus.

\section{EXPERIMENT}

A pulsed YAG pumped dye laser $(5 \mathrm{~ns}, 0.2 \mathrm{~mJ} /$ pulse, 1 $\mathrm{mm}$ radius, $10 \mathrm{~Hz}$ ) frequency doubled to $304.67 \mathrm{~nm}$ was used to dissociate $\mathrm{CH}_{3} \mathrm{I}$ and to ionize the $\mathrm{I}\left({ }^{2} P_{3 / 2}\right)$ fragment by $(2+1)$ resonance-enhanced multiphoton ionization (REMPI). The $1064 \mathrm{~nm}$ fundamental of the Nd:YAG laser $(1-120 \mathrm{~mJ} / \mathrm{pulse}, 3 \mathrm{~mm}$ radius) was used to align the parent molecule prior to dissociation. The UV and IR lasers were counterpropagated into the interaction region. Each laser was focused with a lens $(f=0.20 \mathrm{~m})$ on a pulsed molecular beam of $\mathrm{CH}_{3} \mathrm{I}$ that was diluted in $\mathrm{Ar}(1 \%$, backing pressure $=750$ Torr). The IR beam diameter was larger than the UV laser diameter. The rotational temperature of the $\mathrm{CH}_{3} \mathrm{I}$ is not known precisely but can be estimated from the 7 

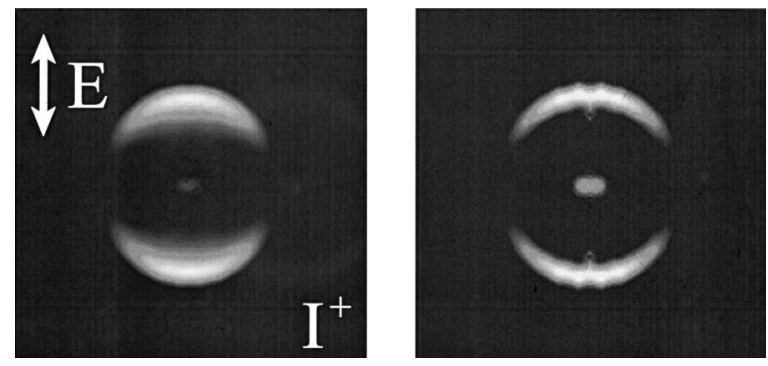

FIG. 1. A raw 2D image (left) and an equatorial slice of a 3D image (right) of $\mathrm{I}\left({ }^{2} P_{3 / 2}\right)$ atoms from the $304.67 \mathrm{~nm}$ dissociation of $\mathrm{CH}_{3} \mathrm{I}$ in the presence of the YAG fundamental $(1064 \mathrm{~nm}, 11 \mathrm{~mJ}, 5 \mathrm{~ns}$ pulse). The direction of the electric vectors of the UV and IR layers are indicated by an arrow.

$\mathrm{K}$ rotational temperature of $\mathrm{NO}$, which was expanded under the same conditions. The following processes occur:

$$
\begin{aligned}
& \mathrm{CH}_{3} \mathrm{I}+h \nu(304.67 \mathrm{~nm}, 0.1 \mathrm{~mJ} / \text { pulse, } 3 \mathrm{~ns}) \\
& \rightarrow \mathrm{CH}_{3}+\mathrm{I}\left({ }^{2} P_{3 / 2}\right), \\
& \mathrm{I}\left({ }^{2} P_{3 / 2}\right)+2 h \nu(304.67 \mathrm{~nm}) \rightarrow \mathrm{I}\left({ }^{4} D_{7 / 2}\right), \\
& \mathrm{I}\left({ }^{4} D_{7 / 2}\right)+h \nu(304.67 \mathrm{~nm}) \rightarrow \mathrm{I}^{+} .
\end{aligned}
$$

The iodine atoms produced by the dye laser were detected by velocity map imaging. ${ }^{11}$ Briefly, the ions were focused onto a microchannel plate $(\mathrm{MCP})$ mounted on the end of a $55 \mathrm{~cm}$ long flight tube. Electrons ejected from the rear plate of the $\mathrm{MCP}$ created an image on a phosphor screen. The image was recorded by a CCD camera and accumulated in a computer. The experimentally observed images were back-projected by a method similar to that used in computerized tomography. ${ }^{12}$ The apparent anisotropy parameter, $\beta$, was obtained by a least squares fit of the 3D slice to the angular distribution function,

$$
I(\theta)=(1 / 4 \pi)\left\{1+\beta P_{2}(\cos \theta)\right\},
$$

where $I(\theta)$ is the normalized angular distribution of the I atom fragment, $\theta$ is the angle between the velocity of the I atom fragment and the electric vector of the dissociation laser beam, and $P_{2}(x)=\left(3 x^{2}-1\right) / 2$.

\section{RESULTS}

A typical image and its back-projection are shown in Fig. 1. Figure 2 shows that the anisotropy parameter $\beta$ increases with IR laser intensity when the IR and UV lasers have parallel polarizations and are simultaneously fired. As the IR laser intensity increases, the angular distribution becomes more anisotropic. This external field effect becomes appreciable for IR laser intensities $>2 \mathrm{~mJ} /$ pulse and reaches an asymptotic value at $10 \mathrm{~mJ} /$ pulse. Above that energy the IR laser causes a decrease in $\mathrm{I}^{+}$signal, although the apparent $\beta$ value remains constant. Our explanation is that the IR laser above a certain intensity does not merely align but also dissociates $\mathrm{CH}_{3} \mathrm{I}$ into a different channel. This new channel is not $\mathrm{CH}_{3}+\mathrm{I}\left({ }^{2} P_{1 / 2}\right)$, because the $\mathrm{I}\left({ }^{2} P_{1 / 2}\right)$ signal is also observed at $305.57 \mathrm{~nm}$ to decrease at IR pulse energies above $10 \mathrm{~mJ}$. The nearly constant $\beta$ value at higher pulse energies is thought to originate from $\mathrm{CH}_{3} \mathrm{I}$ molecules situated further from the center of the region of strongest focus. In other

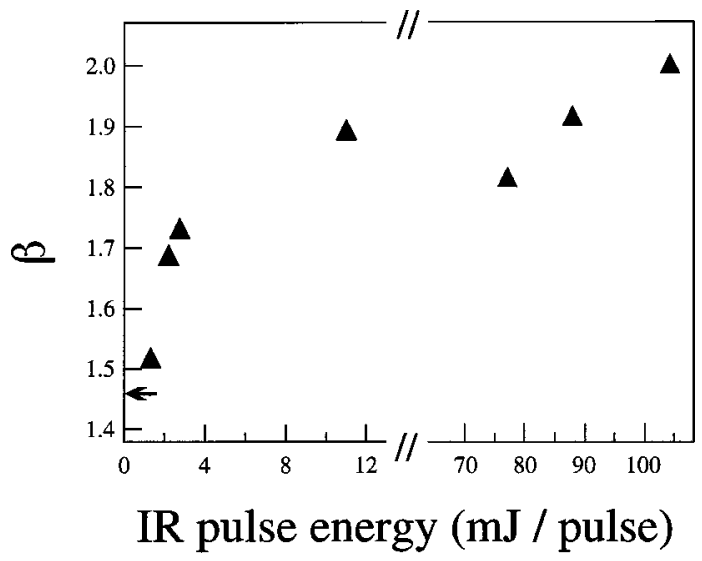

FIG. 2. Change of the anisotropy parameter $\beta$ with $1064 \mathrm{~nm}$ laser intensity for $\mathrm{I}\left({ }^{2} P_{3 / 2}\right)$ photofragments from the photodissociation of $\mathrm{CH}_{3} \mathrm{I}$ at $304 \mathrm{~nm}$. The arrow at zero IR energy indicates a $\beta$ value averaged over several runs. The UV and IR lasers have parallel polarizations.

words, in the volume where one would expect very strong alignment and even localization, the IR laser produces another dissociation channel.

Figure 3 shows the variation of $\beta$ with the delay $\tau$ between the UV and IR (10 mJ/pulse) laser pulses. An appreciable change in $\beta$ is observed when the two laser pulses are temporally overlapped within \pm 2 ns. For $|\tau|>2 \mathrm{~ns}$, the value of $\beta$ was $\sim 1.4$. The average of $\beta$ over several runs in the absence of the IR laser was $1.47 \pm 0.05$, as shown by the arrow in Fig. 2. Figure 4 shows the energy distribution of the $\mathrm{CH}_{3}+\mathrm{I}$ photofragments. No appreciable change is observed in the center-of-mass energy distribution with and without the nonresonant IR laser pulse.

\section{DISCUSSION}

The Hamiltonian for second order perturbation by the electric field due to the polarization anisotropy of the molecule is given by

$$
\begin{aligned}
H= & -\left(E^{2} / 2\right)\left(\alpha_{x x} \sin ^{2} \theta \cos ^{2} \psi+\alpha_{y y} \sin ^{2} \theta \sin ^{2} \psi\right. \\
& \left.+\alpha_{z z} \cos ^{2} \theta\right),
\end{aligned}
$$

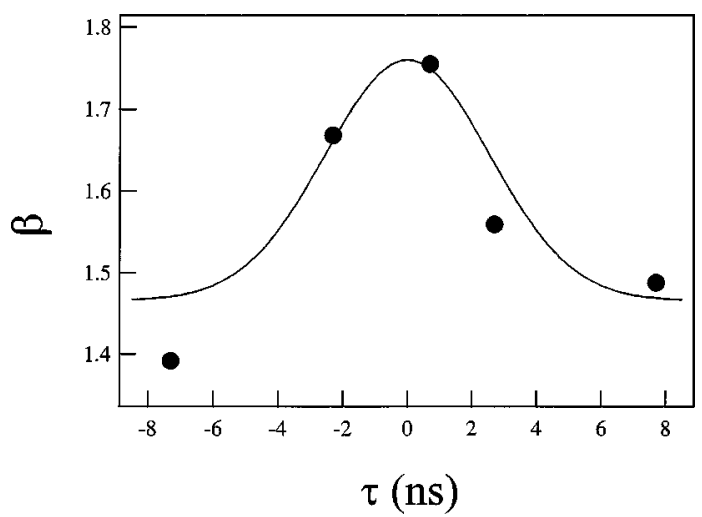

FIG. 3. Temporal profile of $\beta$ values for various delays between the UV laser pulse $(0.1 \mathrm{~mJ} / \mathrm{pulse})$ and the IR laser pulse $(10 \mathrm{~mJ} / \mathrm{pulse})$. For $|\tau|$ $>2 \mathrm{~ns}$, the average value of $\beta$ over several runs, in the absence of the IR laser, is 1.47. The curve shows a temporal overlap of two Gaussian curves (3 ns and $5 \mathrm{~ns}$ width). 


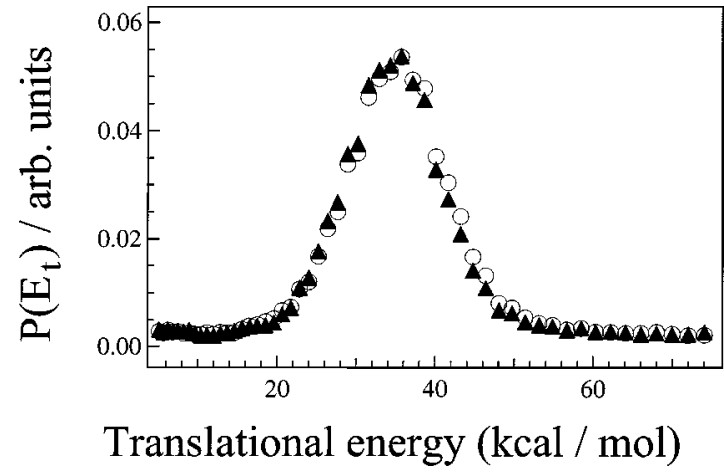

FIG. 4. Center-of-mass transnational energy distribution of photofragments from the photodissociation of $\mathrm{CH}_{3} \mathrm{I}$ at $304.67 \mathrm{~nm}$. The probed photofragment is $\mathrm{I}\left({ }^{2} P_{3 / 2}\right)$. The triangles were measured with the $1064 \mathrm{~nm}$ IR laser pulse $(2-11 \mathrm{~mJ} /$ pulse, $5 \mathrm{~ns}$ duration); the circles were measured without the IR laser pulse.

where $x, y, z$ are the principle axes of the polarizability tensor, $\theta$ is the angle between the $z$ axis and the axis of the electric field, and $\psi$ is an angle of rotation of the molecule about the $z$ axis. The angle $\psi$ is zero when the $x$ axis is perpendicular to the electric field. Equation (2) can be rewritten as

$$
\begin{aligned}
H= & -\left(E^{2} / 2\right)\left\{\alpha+(\eta / 2) \sin ^{2} \theta \cos 2 \psi\right. \\
& \left.+\Delta \alpha\left(3 \cos ^{2} \theta-1\right) / 2\right\},
\end{aligned}
$$

where $\alpha=\left(\alpha_{x x}+\alpha_{y y}+\alpha_{z z}\right) / 3$ is the mean polarizability; $\eta$ $=\alpha_{x x}-\alpha_{y y}$ and $\Delta \alpha=\alpha_{z z}-\alpha$ are anisotropies. For a symmetric top or linear molecule, $\eta=0$.

Using classical statistical mechanics, we calculate the perturbed angular distribution of a molecule with a symmetry axis. The angular distribution is given by

$$
\begin{aligned}
P(\theta)= & \exp \{-H(\theta) / k T\} / \int_{0}^{2 \pi} \int_{0}^{\pi} d \varphi^{\prime} d \theta^{\prime} \sin \theta^{\prime} \\
& \times \exp \left\{-H\left(\theta^{\prime}\right) / k T\right\} .
\end{aligned}
$$

It is convenient to introduce the dimensionless parameter $\gamma$ $=E^{2} \Delta \alpha / 2 k T$. In the weak field approximation this parameter will be small. Equation (4) can now be written compactly as

$$
\begin{aligned}
P(\theta)= & \exp \left\{\gamma P_{2}(\cos \theta)\right\} / \int_{0}^{2 \pi} \int_{0}^{\pi} d \varphi^{\prime} d \theta^{\prime} \sin \theta^{\prime} \\
& \times \exp \left\{\gamma P_{2}\left(\cos \theta^{\prime}\right)\right\} .
\end{aligned}
$$

This expression is valid for any value of $\gamma$, but only small values of $\gamma$ are relevant to our weak field (ns laser) experimental conditions. Expanding Eq. (5) in powers of $\gamma$ and keeping only linear terms, the denominator is $4 \pi$ and the normalized angular distribution is

$$
P(\theta)=(1 / 4 \pi)\left\{1+\gamma P_{2}(\cos \theta)\right\} .
$$

The average value of $\cos ^{2} \theta$ over this angular distribution is $1 / 3+2 \gamma / 15$. The validity of classical statistical mechanics in this system can be tested by considering the rotational constants of $\mathrm{CH}_{3} \mathrm{I}$, i.e., $A=5.11 \mathrm{~cm}^{-1}$ and $B=C=0.25 \mathrm{~cm}^{-1}$. The motion around the threefold axis may be confined to only a few quantum states, but it is irrelevant to the present problem. At $7 \mathrm{~K}$ the most probable value of $J$ is 4 . It is likely that a quantum treatment would change the factor $2 / 15$ somewhat but would not alter the form of Eq. (6). In view of the large uncertainty in $E$, a more exact quantum calculation is not warranted.

For methyl iodide $\alpha=7.54 \times 10^{-24} \mathrm{~cm}^{3}$ and $\Delta \alpha=1.43$ $\times 10^{-24} \mathrm{~cm}^{3}{ }^{13}$ Because the YAG laser that was used was not seeded, inhomogeneities exist in the beam ("hot spots"), and an exact measurement of the strength of the optical field was not possible. Instead the value of $\gamma$ at $3 \mathrm{~mJ}$ pulse energy is determined from the initial slope of Fig. 2 to have a value of 0.28 . Referring to Eq. (6), this means that $\left\langle\cos ^{2} \theta\right\rangle$ increases from $1 / 3$ to $1 / 3+0.037$. In the experiments of Sakai et al. ${ }^{9}$ a much larger increase was observed because their laser intensity was about one hundred times stronger than ours.

Suppose that the angle between the electric fields of the dissociating and aligning lasers is $\chi$. The distribution of molecular axes referred to the electric vector of the dissociating laser is obtained from Eq. (4) by using the spherical harmonic addition theorem and then averaging over azimuthal angles. The result is

$$
P\left(\theta_{s}\right)=(1 / 4 \pi)\left\{1+\gamma P_{2}(\cos \chi) P_{2}\left(\cos \theta_{s}\right)\right\},
$$

where $\theta_{s}$ is the angle between the principle axis of the polarizability tensor and the electric vector of the dissociating laser. Those molecules that absorb light have a distribution of axes given by:

$$
\begin{aligned}
I\left(\theta_{s}\right)= & P\left(\theta_{s}\right)\left\{1+\beta_{0} P_{2}\left(\cos \theta_{s}\right)\right\}=(1 / 4 \pi)\left[1+\left\{\beta_{0}\right.\right. \\
& \left.+\gamma\left(1+2 \beta_{0} / 7-\beta_{0}^{2} / 5\right)\right\} P_{2}(\cos \chi) P_{2}\left(\cos \theta_{s}\right) \\
& \left.+(18 / 35) \beta_{0} \gamma P_{2}(\cos \chi) P_{4}\left(\cos \theta_{s}\right)\right] .
\end{aligned}
$$

For $\chi=0$, the observed anisotropy parameter for $P_{2}\left(\cos \theta_{s}\right), \beta_{\text {eff }}$, is given by

$$
\beta_{\mathrm{eff}}=\beta_{0}+\gamma\left(1+2 \beta_{0} / 7-\beta_{0}^{2} / 5\right) \text {. }
$$

The angular distribution of the I atom photofragments is fitted to Eq. (8) assuming axial recoil. The value of $\beta_{0}$ was previously reported to be $1.5 \pm 0.1$ for a dissociation wavelength of $304 \mathrm{~nm} .{ }^{11,14}$ In the present experiments, the value of $\beta_{0}$ obtained in the absence of the aligning laser is 1.47 \pm 0.05 . The increase of the coefficient of $P_{2}\left(\cos \theta_{s}\right)$ with increasing pulse energy of the aligning IR laser shown in Fig. 2 is fitted by values of $\gamma$, which increase from 0 to 0.28 in the energy range 0 to $4 \mathrm{~mJ}$. From Eq. (9) and the definition of $\gamma$, the value of the rms optical electric field is 5.9 $\times 10^{6} \mathrm{~V} / \mathrm{cm}$ or $4.6 \times 10^{10} \mathrm{~W} / \mathrm{cm}^{2}$ at $4 \mathrm{~mJ}$ pulse energy, which is in agreement with our estimated value of $10^{10}-10^{11} \mathrm{~W} / \mathrm{cm}^{2}$. The coefficient of $P_{4}\left(\cos \theta_{s}\right)$ is so small that similar fitting of $\gamma$ does not give reliable values. It follows from Eq. (7) that, depending on the angle $\chi$ between the $E$ vectors of the two lasers, the IR laser can either increase or decrease the observed anisotropy. When the polarizations were perpendicular to each other, a small decrease in anisotropy was indeed seen.

In principle, strong electric fields can align molecules that have an anisotropic polarizability tensor. We have demonstrated that methyl iodide molecules can be aligned by a 
ns laser. Moreover, by varying the angle between the electric vectors of the aligning and dissociating electric fields within certain limits, one can control the anisotropy of the fragment velocity distribution.

\section{ACKNOWLEDGMENTS}

M.K. thanks the Ministry of Education of Japan for partial support of this work by a Grant-in-Aid in a priority field "Molecular Physical Chemistry.', The research of R.B. and R.J.G. was supported by the U.S. National Science Foundation and the U.S. Department of Energy, respectively.

${ }^{1}$ H. J. Loesch, Annu. Rev. Phys. Chem. 46, 555 (1995).

${ }^{2}$ K. J. Franks, H. Li, S. R. Kuy, and W. Kong, Chem. Phys. Lett. 302, 151 (1999).

${ }^{3}$ B. Friedrich and D. Herschbach, Phys. Rev. Lett. 74, 4623 (1995).
${ }^{4}$ G. R. Kumar, P. Gross, C. P. Safvan, F. A. Rajgara, and D. Mathur, Phys. Rev. A 53, 3098 (1996).

${ }^{5}$ P. Dietrich, D. T. Strickland, M. Laberge, and P. B. Corkum, Phys. Rev. A 47, 2305 (1993).

${ }^{6}$ P. B. Corkum, C. Ellert, M. Mehendale, P. Dietrich, S. Hankin, S. Aseyev, D. Rayner, and D. Villeneuve, Discuss. Faraday Soc. 113, 47 (1999).

${ }^{7}$ T. Seideman, J. Chem. Phys. 106, 2881 (1997).

${ }^{8}$ J. H. Posthumus, J. Plumridge, M. K. Thomas, K. Codling, L. J. Frasinski, A. J. Langley, and P. F. Taday, J. Phys. B. 31, L553 (1998).

${ }^{9}$ H. Sakai, C. P. Sufvan, J. J. Larsen, K. M. Hilligsoe, K. Hald, and H. Stapelfeldt, J. Chem. Phys. 110, 10235 (1999).

${ }^{10}$ J. Larsen, I. Wendt-Larsen, and H. Stapelfeldt, Phys. Rev. Lett. 83, 1123 (1999).

${ }^{11}$ A. T. J. B. Eppink and D. H. Parker, J. Chem. Phys. 109, 4758 (1998).

${ }^{12}$ Y. Sato, Y. Matsumi, M. Kawasaki, K. Tsukiyama, and R. Bersohn, J. Phys. Chem. 99, 16307 (1995).

${ }^{13}$ J. Applequist, J. R. Carl, and K. K. Fung, J. Am. Chem. Soc. 94, 2952 (1972).

${ }^{14}$ J. R. Syage, J. Chem. Phys. 105, 1007 (1996). 